\title{
Critical appraisal of qualitative research - necessity, partialities and the issue of bias
}

Veronika Williams; Anne-Marie Boylan; David Nunan

Veronika Williams, (corresponding author)

Nuffield Department of Primary Care Health Sciences, University of Oxford, Radcliffe Observatory Quarter, Woodstock Road, Oxford, OX2 6GG

veronika.williams@phc.ox.ac.uk

Anne-Marie Boylan

Nuffield Department of Primary Care Health Sciences, University of Oxford, Radcliffe Observatory Quarter, Woodstock Road, Oxford, OX2 6GG

David Nunan

Nuffield Department of Primary Care Health Sciences, University of Oxford, Radcliffe Observatory Quarter, Woodstock Road, Oxford, OX2 6GG

Keywords: Qualitative health research, critical appraisal, patient experience Competing interests: Drs Williams, Boylan and Nunan have no competing interests to disclose.

Contributorship: VW and DN conceived the idea to this article; VW wrote the first draft of the article with $\mathrm{AMB}$ and $\mathrm{DN}$ contributing and revising subsequent drafts. All authors approved the final submitted article.

Acknowledgements: We would like to thank Dr Kamal Mahtani for feedback on an earlier version of this paper.

Funding info: there are no funders to report for this submission

Ethical approval information: not applicable

Data sharing statement: not applicable 


\section{Abstract}

This is the second of three articles in the EBM learning series exploring the role and nature of qualitative research in evidence-based health care. Here we discuss the debate on assessing the rigour of qualitative research and explore the strengths and weakness of existing tools aimed at doing so. We summarise by questioning the idea of a 'unified paradigm' and identify a need for better tools that appropriately assess the rigour of different qualitative methodologies. 


\section{Introduction}

Qualitative evidence allows researchers to analyse human experience and provides useful exploratory insights into experiential matters and meaning, often explaining the 'how' and 'why'. As we have argued previously [1], qualitative research has an important place within evidence-based health care, contributing to amongst other things policy on patient safety [2] prescribing [3, 4], and understanding chronic illness[5]. Equally, it offers additional insight into quantitative studies, explaining contextual factors surrounding a successful intervention or why an intervention might have 'failed' or 'succeeded' where effect sizes cannot. It is for these reasons the MRC strongly recommends including qualitative evaluations when developing and evaluating complex interventions [6].

'Qualitative research' is an umbrella term that refers to various research methodologies including ethnography, phenomenology, action research, discourse analysis and grounded theory, that use textual and/or visual rather than numerical data to explore meaning and perspectives of phenomena. However, it is often considered one unified approach and this lack of methodological distinction is problematic, particularly when thinking about how best to assess rigour in studies using qualitative methods [7-9].

\section{Critical appraisal of qualitative research}

Is it necessary?

Although the importance of qualitative research to improve health services and care is now increasingly widely supported [1] , the role of appraising the quality of qualitative health research is still debated $[8,10]$. Despite a large body of literature focusing on appraisal and rigour [9, 11-15], often referred to as 'trustworthiness' [16] in qualitative research, there remains debate about how to - and even whether to - critically appraise qualitative research [8-10, 17-19]. However, if we are to argue that qualitative research is integral to evidencebased health care, then any argument to omit a crucial element of evidence-based practice is difficult to justify. That being said, simply applying the standards of rigour used to appraise studies based on the positivist paradigm ${ }^{1}$, would be misplaced given the different epistemological underpinnings of the two types of data.

Given its scope and its place within health research, the robust and systematic appraisal of qualitative research to assess its trustworthiness, is as paramount to its implementation in clinical practice as any other type of research. It is important to appraise different qualitative studies in relation to the specific methodology used because the methodological approach is linked to the 'outcome' of the research (eg theory development,

\footnotetext{
${ }^{1}$ Positivism depends on quantifiable observations to test hypotheses and assumes that the researcher is independent from the study. Research situated within a positivist paradigm is based purely on facts and consider the world to be external and objective and is concerned with validity, reliability and generalisability as measures of rigour.
} 
phenomenological understandings, credibility of findings). Moreover, appraisal needs to go beyond merely describing the specific details of the methods used (e.g. how data were collected and analysed), with additional focus needed on the overarching research design and its appropriateness in accordance with the study remit and objectives.

Poorly conducted qualitative research has been described as "worthless, becomes fiction, and loses its utility" [20]. But without a deep understanding of concepts of quality in qualitative research, or at least an appropriate means to assess its quality, good qualitative research also risks being dismissed, particularly in the context of evidence-based health care where end users may not be well-versed in this paradigm.

\section{How is appraisal currently performed?}

Appraising the quality of qualitative research is not a new concept - there are a number of published appraisal tools, frameworks and checklists in existence [8, 21-23]. An important and often overlooked point is the confusion between tools designed for appraising methodological quality and reporting guidelines designed to assess the quality of methods reporting. An example is the Consolidate Criteria for Reporting Qualitative Research (COREQ) [24] checklist, which was designed to provide standards for authors when reporting qualitative research but is often mistaken for a methods appraisal tool [10].

Broadly speaking there are two types of critical appraisal approaches for qualitative research: checklists and frameworks. Checklists have often been criticised for confusing quality in qualitative research with 'technical fixes' [21,25], resulting in the erroneous prioritisation of particular aspects of methodological processes over others (e.g. multiple coding, triangulation). It could be argued that a checklist approach adopts the positivist paradigm, where the focus is on objectively assessing 'quality' where the assumptions is that the researcher is independent from the research conducted. This may result in the application of quantitative understandings of bias in order to judge aspects of recruitment, sampling, data collection and analysis in qualitative research papers. One of the most widely used appraisal tools is the Critical Appraisal Skills Programme (CASP) [26], and along with the JBI QARI (Joanna Briggs Institute Qualitative Assessment and Assessment Instrument), [27], presents examples which tend to mimic the quantitative approach to appraisal. The CASP qualitative tool follows that of other CASP appraisal tools for quantitative research designs developed in the 1990s. The similarities are therefore unsurprising given the status of qualitative research at that time.

Frameworks focus on the overarching concepts of quality in qualitative research, including transparency, reflexivity, dependability, transferability (see Box 1) [11-13, 15, 16, 20, 28]. However, unless the reader is familiar with these concepts - their meaning and impact, and how to interpret them - they will have difficulty applying them when critically appraising a paper. 
The main issue concerning currently available checklist and framework appraisal methods is that they take a broad brush approach to 'qualitative' research as whole, with few, if any, sufficiently differentiating between the different methodological approaches (e.g. Grounded Theory, Interpretative Phenomenology, Discourse Analysis) nor different methods of data collection (interviewing, focus groups, observations). In this sense, it is akin to taking the entire field of 'quantitative' study designs and applying a single method or tool for their quality appraisal. In the case of qualitative research, checklists therefore offer only a blunt and arguably ineffective tool and potentially promote an incomplete understanding of good 'quality' in qualitative research. Likewise, current framework methods do not take into account how concepts differ in their application in across the variety of qualitative approaches and, like checklists, they also do not differentiate between different qualitative methodologies.

\section{On the need for specific appraisal tools}

Current approaches to appraisal of the methodological rigour of the differing types of qualitative research converge towards checklists or frameworks. More importantly, the current tools do not explicitly acknowledge the prejudices that may be present in the different types of qualitative research.

It is important to note that we are not referring to these prejudices as 'biases', as to do so is to invoke quantitative understandings of bias which are not wholly compatible with qualitative research $[29,30]$. To avoid confusion, we will refer to them as partialities. These partialities do not relate to reliability, validity and reproducibility (the quantitative concepts of rigour) but rather to transferability, credibility, reflexivity and dependability (see box 1). However we often talk about these concepts in general terms and it might be helpful to give some explicit examples of how the 'technical processes' affect these. For example, partialities related to:

- Selection: recruiting participants via gatekeepers, such as health care professionals or clinicians, who may select them based on whether they believe them to be 'good' participants for interviews/ focus groups;

- Data collection: poor interview guide with closed questions which encourage yes/no answers and/ leading questions;

- Reflexivity and transparency: where researchers may focus their analysis on preconceived ideas rather than ground their analysis in the data and do not reflect about the impact of this in a transparent way.

The lack of tailored, method specific appraisal tools has potentially contributed to the poor uptake and use of qualitative research for informing evidence-based decision making. To improve upon this situation, we propose the need for more robust quality appraisal tools that explicitly encompass both the core design aspects of all qualitative research (sampling/ 
data collection/ analysis) but also considered the specific partialities that can be presented with different methodological approaches. Such tools might draw on the strengths of current frameworks and checklists, whilst providing users with sufficient understanding of concepts of rigour in relation to the different types of qualitative methods. We provide an outline of such tools in the third and final paper in this series.

\section{Conclusion}

As qualitative research becomes ever more embedded in health science research, and in order for that research to better impact on health care decisions, we need to rethink critical appraisal and develop tools that allow differentiated evaluations of the myriad of qualitative methodological approaches rather than continuing to treat qualitative research as a single unified approach. 
Box 1: Concepts of rigour or trustworthiness within qualitative research[31]

Transferability: the extent to which the presented study allows readers to make connections between the study's data and wider community settings, i.e., transfer conceptual findings to other contexts.

Credibility: extent to which a research account is believable and appropriate, particularly in relation to the stories told by participants, and the interpretations made by the researcher.

Reflexivity: refers to the researchers' engagement of continuous examination and explanation of how they have influenced a research project from choosing a research question to sampling, data collection, analysis and interpretation of data.

Transparency: making explicit the whole research process from sampling strategies, data collection to analysis. Rationale for decisions made are as important as the decisions themselves. 
REFERNCES:

1. Williams, V., A.-M. Boylan, and D. Nunan, Qualitative research as evidence: expanding the paradigm for evidence-based healthcare. BMJ Evidence-Based Medicine, 2019: p. bmjebm-2018-111131.

2. Lingard, L., et al., Evaluation of a preoperative checklist and team briefing among surgeons, nurses, and anesthesiologists to reduce failures in communication. Archives of surgery, 2008. 143(1): p. 12-17.

3. Carthy, P., et al., A study of factors associated with cost and variation in prescribing among GPs. Family Practice, 2000. 17(1): p. 36-41.

4. Coenen, S., et al., Antibiotics for coughing in general practice: a qualitative decision analysis. Family practice, 2000. 17(5): p. 380-385.

5. Bury, M., Chronic illness as biographical disruption. Sociology of health \& illness, 1982. 4(2): p. 167-182.

6. Moore, G.F., et al., Process evaluation of complex interventions: Medical Research Council guidance. bmj, 2015. 350: p. h1258.

7. Rolfe, G., Validity, trustworthiness and rigour: quality and the idea of qualitative research. Journal of advanced nursing, 2006. 53(3): p. 304-310.

8. Smith, B. and K.R. McGannon, Developing rigor in qualitative research: Problems and opportunities within sport and exercise psychology. International review of sport and exercise psychology, 2018. 11(1): p. 101-121.

9. Dixon-Woods, M., et al., The problem of appraising qualitative research. Quality and Safety in Health Care, 2004. 13(3): p. 223-225.

10. Majid, U. and M. Vanstone, Appraising qualitative research for evidence syntheses: a compendium of quality appraisal tools. Qualitative health research, 2018: p. 1049732318785358.

11. Greenhalgh, T. and R. Taylor, How to read a paper: Papers that go beyond numbers (qualitative research). BMj, 1997. 315(7110): p. 740-743.

12. Kuper, A., L. Lingard, and W. Levinson, Critically appraising qualitative research. Bmj, 2008. 337(aug07_3): p. a1035-a1035.

13. Mays, N. and C. Pope, Qualitative research: rigour and qualitative research. Bmj, 1995. 311(6997): p. 109-112.

14. Dennison, L., et al., Opportunities and challenges for smartphone applications in supporting health behavior change: qualitative study. Journal of medical Internet research, 2013. 15(4).

15. Yardley, L., Demonstrating validity in qualitative psychology. Qualitative psychology: A practical guide to research methods, 2008. 2: p. 235-251.

16. Guba, E.G. and Y.S. Lincoln, Epistemological and methodological bases of naturalistic inquiry. ECTJ, 1982. 30(4): p. 233-252.

17. Garside, R., Should we appraise the quality of qualitative research reports for systematic reviews, and if so, how? Innovation: The European Journal of Social Science Research, 2014. 27(1): p. 67-79.

18. Garside, R., Systematic review and sythesis of qualitative research, in Understanding and Using Health Experiences - Improving patient care, S. Ziebland, et al., Editors. 2013, Oxford University Press: Oxford.

19. Long, $\mathrm{T}$. and $\mathrm{M}$. Johnson, Rigour, reliability and validity in qualitative research. Clinical effectiveness in nursing, 2000. 4(1): p. 30-37.

20. Morse, J.M., et al., Verification strategies for establishing reliability and validity in qualitative research. International journal of qualitative methods, 2002. 1(2): p. 13-22.

21. Hannes, K., C. Lockwood, and A. Pearson, A Comparative Analysis of Three Online Appraisal Instruments' Ability to Assess Validity in Qualitative Research. Qualitative Health Research, 2010. 20(12): p. 1736-1743. 
22. Harris, J., Supplementary guidance for inclusion of qualitative research in Cochrane systematic reviews of interventions. 2011.

23. Santiago-Delefosse, M., et al., Quality of qualitative research in the health sciences: Analysis of the common criteria present in 58 assessment guidelines by expert users. Social Science \& Medicine, 2016. 148: p. 142-151.

24. Tong, A., P. Sainsbury, and J. Craig, Consolidated criteria for reporting qualitative research (COREQ): a 32-item checklist for interviews and focus groups. International Journal for Quality in Health Care, 2007. 19(6): p. 349-357.

25. Barbour, R.S., Checklists for improving rigour in qualitative research: a case of the tail wagging the dog? BMJ: British Medical Journal, 2001. 322(7294): p. 1115.

26. CASP (Critical Appraisal Skills Programme). date unknown, [http://www.phru.nhs.uk/Pages/PHD/CASP.htm].

27. Institute, J.B., JBI QARI Critical appraisal checklist for interpretive \& critical research. The Joanna Briggs Institute, Adelaide, 2014.

28. Guba, E.G. and Y.S. Lincoln, Competing paradigms in qualitative research. Handbook of qualitative research, 1994. 2(163-194): p. 105.

29. Thorne, S., J. Stephens, and T. Truant, Building qualitative study design using nursing's disciplinary epistemology. Journal of Advanced Nursing, 2016. 72(2): p. 451-460.

30. Galdas, P., Revisiting Bias in Qualitative Research: Reflections on Its Relationship With Funding and Impact. 2017, SAGE Publications Sage CA: Los Angeles, CA.

31. Given, L.M., The Sage encyclopedia of qualitative research methods. 2008: Sage Publications. 